\title{
Carcinosarcoma of the Uterine Corpus with Alpha-Fetoprotein-Producing Hepatoid Adenocarcinoma: A Report of Two Cases
}

\author{
Ryuji Kawaguchi Naoto Furukawa Yoshihiko Yamada \\ Hidekazu Ooi Hiroshi Kobayashi \\ Department of Obstetrics and Gynecology, Nara Medical University, Kashihara, Japan
}

\section{Key Words}

Uterine carcinosarcoma $\cdot$ Hepatoid adenocarcinoma $\cdot$ Rhabdomyosarcoma

Alpha-fetoprotein

\begin{abstract}
We report two cases of uterine carcinosarcoma associated with alpha-fetoprotein (AFP)producing hepatoid adenocarcinoma. Samples were obtained from two women aged 63 and 82 years. Serum AFP levels of the two samples were 10,131 and $401 \mathrm{ng} / \mathrm{ml}$, respectively. Histologically, in both cases the tumor cells were composed of hepatoid adenocarcinoma component and sarcoma component including rhabdomyosarcoma. Immunohistochemical analyses revealed that AFP was expressed in the cytoplasm of the carcinomatous component. After surgery, the patients received six courses of carboplatin/paclitaxel chemotherapy, and the serum levels of AFP decreased to normal range. The first patient is alive and well at the 2-year follow-up, while the second patient died of disease 1 year after initial operative treatment. This is, to our knowledge, the second report of carcinosarcoma of the uterine corpus with AFP-producing hepatoid adenocarcinoma, as proven by immunohistochemical analyses.
\end{abstract}

\section{Introduction}

Hepatoid adenocarcinomas are tumors that arise outside the liver but which resemble hepatic tissue and produce alpha-fetoprotein (AFP). Several reports on this subject have been found in the literature. Hepatoid adenocarcinomas have been identified in many sites, including the stomach, lung, rectum, renal pelvis, ovary, uterus, and vagina. Until now, only one case of hepatoid adenocarcinoma of uterine corpus carcinosarcoma and elevated serum AFP has been reported. 
We report two cases of AFP-producing hepatoid adenocarcinomas of uterine corpus carcinosarcoma with rhabdoid differentiation, and discuss the pathologic features and clinical significance of this pattern of differentiation in uterine corpus tumors.

\section{Case Reports}

\section{Case 1}

A 63-year-old multiparous woman, gravida 4, para 3, complained of abnormal vaginal bleeding of 2 months' duration. She went into menopause at age 54 . The patient had no remarkable medical or family histories. On vaginal examination, the uterus was enlarged, non-tender, smooth and movable. Vaginal ultrasonogram and magnetic resonance imaging showed a polypoid mass of $14 \times 10 \mathrm{~cm}$ in size in the uterus, possibly representing a carcinosarcoma or leiomyosarcoma. No free pelvic fluid was identified. Computed tomography (CT) showed the liver and bile ducts to be normal. The endometrial Pap smear was evaluated as positive. Biopsies of the endometrium were carried out and the histological diagnosis revealed carcinosarcoma. The serum AFP level was 10,131 ng/ml (normal $<20$ ). Other tumor markers and liver function test results were within normal limits. The patient underwent a total abdominal hysterectomy, bilateral salpingo-oophorectomy and pelvic lymphadenectomy.

The resected enlarged uterus, weighing $560 \mathrm{~g}$ and measuring $14 \times 12 \times 7 \mathrm{~cm}$, had a smooth glistening surface. The polypoid tumor originated from the uterine cavity when the anterior wall of the uterus was opened. The uterine adnexae were normal in shape and size. There was no evidence of a tumorous lesion. Microscopic examination showed that the polypoid tumor was a mixture of carcinomatous and sarcomatous components (ig. 1a). The tumor had invaded the uterine cervix, but no metastases were found in the dissected lymph nodes. The carcinomatous component showed adenocarcinoma arranged in sheets and cords with a hepatoid appearance. Reactivity for AFP was cytoplasmic in the hepatoid carcinoma lesion (fig. 1b). The sarcomatous component showed no reactivity for AFP. The large pleomorphic cells were positive for desmin, actin, CD10, MyoD1, and myoglobin and could be regarded as being rhabdomyosarcoma. There was no staining with S-100. In summary, the tumor was histopathologically diagnosed as heterologous uterine corpus carcinosarcoma associated with AFPproducing hepatoid adenocarcinoma.

Postoperatively, the patient received six courses of chemotherapy with paclitaxel $\left(175 \mathrm{mg} / \mathrm{m}^{2}\right)$ and carboplatin (AUC 5). This regimen was repeated every 3 weeks. After the third course of chemotherapy, the patient's serum AFP level decreased to $4.5 \mathrm{ng} / \mathrm{ml}$. The patient is alive with no evidence of recurrence or increase in the serum AFP level in the 2 years since treatment.

\section{Case 2}

An 82-year-old woman, gravida 0 , para 0 , was referred to our hospital with vaginal bleeding of 2 months' duration. She had undergone surgery due to breast cancer at the age of 68 . On pelvic examination, enlarged uterus without a palpable adnexal mass was detected. The vaginal wall showed no abnormalities. Portio vaginalis could not be detected, so the endometrial Pap smear could not be done. Magnetic resonance imaging revealed an enhanced mass, $8 \mathrm{~cm}$ in size, in the uterine corpus cavity, possibly representing malignant tumor. CT scan demonstrated ascites and no lymph nodes were detected. CT and ultrasonography revealed no abnormalities in the liver or bile duct. The serum AFP level was elevated (401 ng/ml), but no elevation of other markers, such as carcinoembryonic antigen or carbohydrate antigen 125, was observed. The blood count and serum biochemical data were unremarkable. From these results, malignant uterine tumor was highly suspected. A total abdominal hysterectomy with bilateral salpingo-oophorectomy and pelvic washings was carried out. The resected uterus was enlarged and filled with a spongy material. There was no apparent extrauterine extension of the tumor. A partially necrotic polyp, $8 \mathrm{~cm}$ in size, was present within the endometrial cavity, which infiltrated into the myometrium for about two-thirds of its thickness but did not invade the cervix. Cytology of the pelvic washings was negative. Histologically, the tumor was composed of carcinomatous and sarcomatous components (ig. $2 \mathrm{a}$ ). The carcinomatous component was an endometrioid 
adenocarcinoma with trabecular hepatoid cells, mostly showing hepatocellular carcinoma-like proliferation. Immunohistochemistry also revealed AFP-positivity in many tumor cells (fig. 2b).

The sarcomatous component showed rhabdoid features including spindle-shaped tumor cells. Occasional cells with eosinophilic cytoplasm and cytoplasmic cross-striations were identified. Immunohistochemistry showed focal positivity of tumor cells with desmin, MyoD1, and CD10. On pathologic examination, the tumor was interpreted as being a heterologous uterine corpus carcinosarcoma associated with AFP-producing hepatoid adenocarcinoma.

We did not carry out any adjuvant chemotherapy or irradiation because of the patient's advanced age, in addition, she and her family refused these therapies. Two months after the surgery, the patient's serum AFP level decreased to a normal level. However, multiple lung metastases with a high-serum AFP level reappeared and the patient died 1 year after the operation.

\section{Discussion}

Ishikura et al. [1] proposed the term 'hepatoid adenocarcinoma' to describe a distinct histological subtype of adenocarcinoma. They defined hepatoid adenocarcinoma as AFPproducing carcinoma with a mixture of tubular or papillary adenocarcinoma, with abundant, eosinophilic cytoplasm and centrally located nuclei. Hepatoid adenocarcinoma has some histological features that resemble hepatocellular carcinoma. Hepatoid adenocarcinoma has mostly been found in the stomach, but also occurs in many other organs, including the uterus and ovaries. AFP-producing tumors other than hepatoma and germ cell tumors have been widely reported, especially in hepatoid adenocarcinoma. In uterine corpus carcinosarcoma, however, production of AFP is extremely rare. To our knowledge, six cases of hepatoid adenocarcinoma of the uterine corpus have been reported in the literature. Four cases were categorized as endometrial carcinoma [2-5] and two as carcinosarcoma [6,7]. Microscopically, in both our cases, the tumor cells were composed of hepatoid adenocarcinoma and sarcoma components including rhabdomyosarcoma. Our two cases are also examples of hepatoid adenocarcinomas of uterine corpus carcinosarcoma, because of the close resemblance of the neoplastic cells to hepatoid cells and because of the AFP production. In the present cases, AFP was diffusely localized to the cytoplasm. Judging from the changes in serum AFP values, the AFP content of the tumor and the immunohistochemical localization of AFP in the tumor tissue, AFP was produced by the tumor. In our cases, a close correlation was noted between shrinkage of the pelvic tumor and declining AFP levels. Serum AFP was a good marker for monitoring response to treatment in our patients.

Rhabdomyosarcoma is a sarcoma showing evidence of skeletal or striated muscle differentiation. The World Health Organization classification of uterine mesenchymal tumors places rhabdomyosarcoma in the category of mesenchymal tumors composed of heterologous elements [8]. We consider that immunohistochemical support is necessary for a definitive diagnosis of rhabdomyosarcoma. In our cases, desmin was positive. It is a muscle marker and does not help distinguish between a rhabdomyosarcoma and a leiomyosarcoma. However, MyoD1 and myoglobin (both skeletal muscle markers) were focally positive. This provides unequivocal evidence that the two neoplasms in this report exhibit skeletal muscle differentiation. The sarcomatous lesion included rhabdomyosarcoma with spindle cells and large pleomorphic cells. To date, four cases of uterine carcinosarcoma with rhabdoid features have been reported $[6,9,10]$. All four patients were initially treated surgically with subsequent chemotherapy. In the three cases where clinical follow-up was available, two patients died of disease [9]. 
Carcinosarcoma of the uterine corpus is a rare and aggressive neoplasm that contains both carcinomatous and sarcomatous histologic elements. Carcinosarcoma of the uterine corpus is characterized by a high incidence of advanced stage at diagnosis and high recurrence rates after local therapy. Standard treatment for early-stage patients is hysterectomy and surgical staging. When advanced or recurrent disease is diagnosed, controlling it is difficult and chemotherapy is minimally successful. Some drugs that have been examined as single-agent therapy options are as follows: adriamycin, ifosfamide, cisplatin, and paclitaxel. Paclitaxel is a novel antineoplastic agent derived from the bark of the Pacific yew tree, Taxus brevifolia. The Gynecologic Oncology Group has tested paclitaxel in a variety of studies and has reported activity in ovarian cancer and adenocarcinoma of the endometrium, as well as in squamous and nonsquamous cancers of the cervix. The combination of paclitaxel and carboplatin demonstrated higher activity than that found in previous studies in patients with advanced or recurrent uterine carcinosarcoma [11]. In our first case, adjuvant chemotherapy comprising paclitaxel and carboplatin was administered after surgery, and 2 years after the end of treatment, the patient is still disease-free.

We described two cases of carcinosarcoma of the uterine corpus with AFP-producing hepatoid adenocarcinoma. Further studies with clinical follow-up are necessary to determine the best treatment modalities.

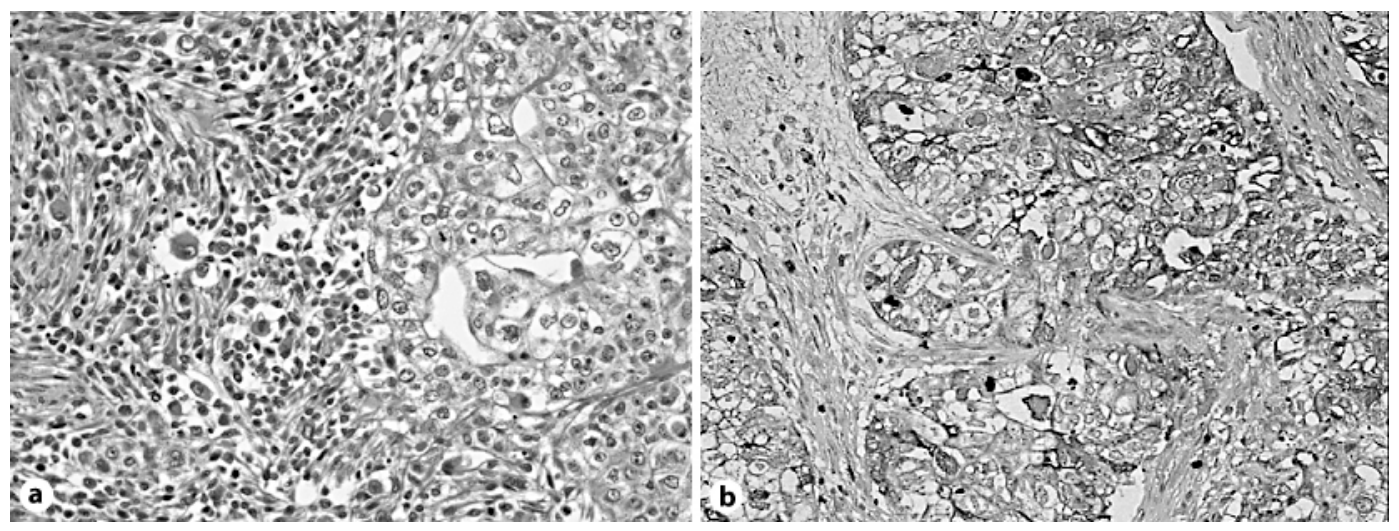

Fig. 1. a Histological appearance of the carcinosarcoma. The carcinomatous component is composed of hepatoid adenocarcinoma and the sarcomatous component of spindle cells and large pleomorphic cells (HE, $\times 400)$. b Tumor cells in the hepatoid areas show immunoreactivity for AFP (AFP, ×400). 

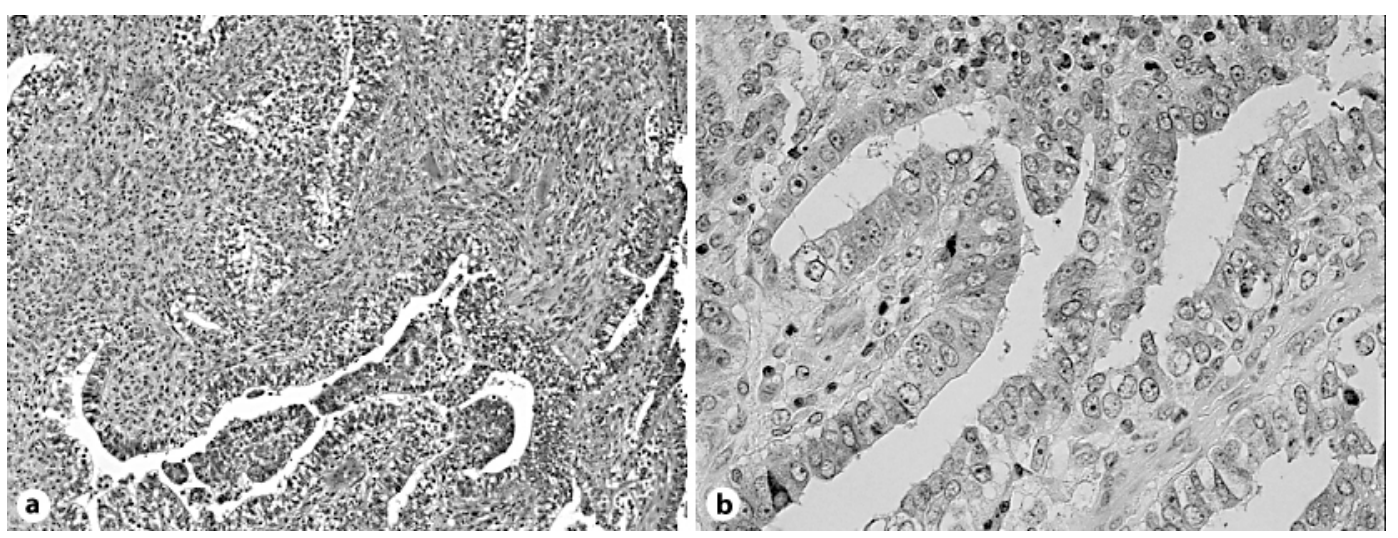

Fig. 2. a Tumor showing a mixture of carcinomatous and sarcomatous components $(\mathrm{HE}, \times 100)$.

b Tumor cells in the hepatoid areas immunoreactive for AFP (AFP, $\times 400)$.

\section{References}

1 Ishikura H, Fukasawa Y, Ogasawara K, Natori T, Tsukada Y, Aizawa M: An AFP-producing gastric carcinoma with features of hepatic differentiation. A case report. Cancer 1985;56:840-848.

- Hoshida Y, Nagakawa T, Mano S, Taguchi K, Aozasa K: Hepatoid adenocarcinoma of the endometrium associated with alpha-fetoprotein. Int J Gynecol Pathol 1996;15:266-269.

-3 Yamamoto R, Ishikura H, Azuma M, Hareyama H, Makinoda S, Koyama Y, Nishi S, Fujimoto S: Alphafetoprotein production by a hepatoid adenocarcinoma of the uterus. J Clin Pathol 1996;49:420-422.

$\checkmark 4$ Toyoda H, Hirai T, Ishii E: Alpha-fetoprotein producing uterine corpus carcinoma: a hepatoid adenocarcinoma of the endometrium. Pathol Int 2000;50:847-852.

5 Adam SF, Yamada SD, Montag A, Rotmensch J: An alpha-fetoprotein-producing hepatoid adenocarcinoma of the endometrium. Gynecol Oncol 2001;83:847-852.

6 Takano M, Shibasaki T, Sato K, Aida S, Kikuchi Y: Malignant mixed Müllerian tumor of the uterine corpus with alpha-fetoprotein-producing hepatoid adenocarcinoma component. Gynecol Oncol 2003;91:444-448.

7 Takahashi Y, Inoue T: Hepatoid carcinoma of the uterus that collided with carcinosarcoma component. Gynecol Oncol 2003;53:323-326.

8 Scully RE, Bonfiglio T, Kurman RJ, Silverberg SG, Wilkinson EJ: Histological Typing of Female Genital Tract Tumors. ed 2, Berlin, Springer Verlag, 1994.

-9 Mount SL, Lee KR, Taatjes DJ: Carcinosarcoma (malignant mixed Müllerian tumor) of the uterus with a rhabdoid tumor component. An immunohistochemical, ultrastructural, and immunoelectron microscopic case study. Am J Clin Pathol 1995;103:235-239.

10 Dmitry YB, Theodore HN, Lynne AE, Wendy LF: Malignant mixed Müllerian tumor with rhabdoid features: A case report of two cases and a review of the literature. Gynecol Oncol 1999;73:145-150.

-11 Toyoshima M, Akahira J, Matsunaga G, Niikura H, Ito K, Yaegashi N, Tase T: Clinical experience with combination paclitaxel and carboplatin therapy for advanced or recurrent carcinosarcoma of the uterus. Gynecol Oncol 2004;94:774-778. 